\title{
The Muddle of Immune Response in Coronavirus Disease-19
}

\author{
Vyas AK*, Garg G and Gupta P
}

Department of Microbiology, All India Institute of Medical Sciences, Bhopal, India

*Corresponding author: Dr Ashish Kumar Vyas, Inspire Faculty, Department of Microbiology, All India Institute of Medical Sciences, Bhopal, India, Email: a88 ashish@yahoo.co.in

\section{Mini Review \\ Volume 5 Issue 3}

Received Date: September 21, 2020

Published Date: October 03, 2020

DOI: $10.23880 /$ oajmb-16000171

\section{Abstract}

Coronavirus Disease-19 (COVID19) caused by the enormously spreadable SARS- CoV-2 virus has appeared as a global pandemic and leads to high mortalities. Approximately 31,243,339 people have been infected so far with this disease which has led to the death of more than 965,103 patients as of 21st Sept 2020. There are many drug molecules under-trials are in phase I and II. The numerous possible candidates for vaccine development against this infectious virus are also in the pipeline. Although, so far no molecule as a therapeutics or vaccine for prevention has been approved. Recent reports have observed that severely ill patients have a differential immunological profile compared to mild COVID-19 infection. Current studies globally observed that the cytokine storm maybe leads to the severity of COVID19 infection. In this article, our focus is to describe the present knowledge and status of differential immune profile among patients infected with COVID19 infection and their association with disease progression mild to severe.

Keywords: Coronavirus Disease-19; T and B cells; Monocytes; Macrophages and Neutrophils

Abbreviations: COVID19: Coronavirus Disease-19; SARS: Severe Acute Respiratory Syndrome; MERS: MiddleEast respiratory syndrome; ESR: Erythrocyte Sedimentation Rate; CRP: C-reactive protein; IL6: Interleukin 6; ARDS: Acute Respiratory Distress Syndrome.

\section{Introduction}

Along with viral infectivity understanding the status immunity of individuals and their association with manifestation of mild or severe symptoms could be crucial to containing the human costs of this pandemic. To describe in summary there are 2 types of immunity induced in response to viral infection. The first line is innate means natural immunity and Adaptive immunity. Natural immunity is known as innate immunity, which is rapid, non-specific and does not have a memory. Innate immunity mediated by dendritic cell, NK cell, monocytes, macrophages and neutrophils and the Adaptive immunity is regulated by lymphocytes ( $\mathrm{T}$ and
B cells). The adaptive immunity has an advantage due to they have specificity and memory development capability.

\section{Immune Response in COVID19}

The immunological response of COVID19 infected patients found to be similar and consistent with those seen in the previous SARS and MERS infections such as increased total neutrophil count and decreased lymphocyte count are common features of all three syndromes [1]. Moreover, a cytokine storm comprising of high levels of inflammatory cytokines is observed in COVID19 infected patients and is correlated with increased severity of the disease [2,3]. The measurement of immune responses along with clinical characteristics can be used for differential diagnosis of COVID patients and for investigation of association with disease severity.

Previously, Cui et al. had found that the incidence of 


\section{Open Access Journal of Microbiology \& Biotechnology}

lymphopenia ( $84 \%$ of patients), and decrease in immune cells such as CD4 T (100\% of patients), CD8 T (87\%), B cells (76\%), and NK (55\%) in SARS patients. Similar to these observations, Wang et al. also found that severe cases of COVID19 were found to be associated with a decreased level of lymphocytes compared to mild cases $[4,5]$. Although the number of CD8 $\mathrm{T}$ cells establishes to be negatively related to the clinical indicators such as Erythrocyte Sedimentation Rate (ESR), C-reactive protein (CRP), and Interleukin 6 (IL6). Other than the number of immune cells, additionally, patients of COVID19 had impaired functionality such as low NK, CD8 T cell secreting IFN-y, and IL-2 compared to healthy controls. Patients with COVID19 also shows lower number of CD107a+,CD8+, CD107a+ NK cells compared to healthy controls [6]. One more recent report by Guang Chen and co-authors found the same and validate that these patients exhibited defective functionality of these lymphocytes in addition to lymphopenia [7].

On the basis of available knowledge, it is clear now that the change in the number of immune cells, particularly CD8 $\mathrm{T}$ cells, could be a reliable predictor of disease progression and disease severity in COVID-19 patients [8]. Presently, no strong and effective treatment strategies or vaccines are accessible for the protection or prevention of the disease. Directing the immune cell number and functionality could be an encouraging strategy for emergent an effective treatment or even a vaccine against this virus. Recent reports well established that other than immune cell functionality and number cytokines level found to be directly associated with disease severity. Numerous studies have found that the IL-6 level is important in the pathogenesis of COVID19 and can predict the severity of the disease. Recently a clinical trial using Tocilizumab drug (ChiCTR2000029765) to block the IL-6 receptor has been on-going to examine the opportunity of IL- 6 as a feasible aim of intervention for COVID19 patients that can moderate the adverse effects of the infection [9]. Tocilizumab is recognized to bind to both membrane-bound and soluble IL- 6 receptors thus important to the inhibition of downstream signal transduction. The study by Zhang C, et al. found that Tocilizumab had improved effectiveness for the treatment of severely ill patients infected with COVID19 [10]. Though, this study was accompanied by relatively small sample size and hence, larger studies are essential to validate this treatment.

\section{Recommendations}

The impression of any widespread infection could be determined by three factors: 1 . the infectivity-virulence of the pathogen, 2. Host Immunity, and 3. the environment or geographical favourability of the pathogen. Although the virulence and transmission of the COVID19 are depended upon its characteristic or nature and cannot be regulated. The handling of the environment emanates with economic and social restrictions, the finest approach to contain the injury caused by the virus would be to target the host immune responses. Nevertheless, this would necessitate a stronger understanding of the immunological outlines of diseased individuals and the discrepancy immune function among asymptomatic, mildly diseased, severely diseased, and fatal individuals. Recognizing key alterations in these profiles might help in narrowing down aims of intervention such as lymphocytes and cytokines. Henceforth, mechanistic studies of aspects associated with alteration in lymphocyte counts, specifically T lymphocytes and NK cells, as well as the higher in cytokine levels in severely ill patients is essential for the supervision of COVID-19 patients.

\section{References}

1. Prompetchara E, Ketloy C, Palaga T (2020) Immune responses in COVID-19 and potential vaccines: Lessons learned from SARS and MERS epidemic. Asian Pac J Allergy Immunol 38(1): 1-9.

2. Ye Q, Wang B, Mao J (2020) The pathogenesis and treatment of the 'Cytokine Storm' in COVID-19. J Infect 80(6): 607-613.

3. Sun X, Wang T, Cai D, Hu Z, Chen J, et al. (2020) Cytokine storm intervention in the early stages of COVID-19 pneumonia. Cytokine Growth Factor Rev 53: 38-42.

4. Wong RS, Wu A, To KF, Lee N, Lam CW, et al. (2003) Haematological manifestations in patients with severe acute respiratory syndrome: retrospective analysis. BMJ 326(7403): 1358-1362.

5. Peiris JSM, Lai ST, Poon L, Guan Y, Yam L, et al. (2003) Coronavirus as a possible cause of severe acute respiratory syndrome. Lancet 361(9366): 1319-1325.

6. Zheng M, Gao Y, Wang G, Song G, Liu S, et al. (2020) Functional exhaustion of antiviral lymphocytes in COVID-19 patients. Cell Mol Immunol 17(5): 533-535.

7. Chen G, Wu D, Guo W, Cao Y, Huang D, et al. (2019) Clinical and immunologic features in severe and moderate Coronavirus Disease 2019. J Clin Invest 130(5): 26202629.

8. Ruan Q Yang K, Wang W, Jiang L, Song J (2020) Clinical predictors of mortality due to COVID-19 based on an analysis of data of 150 patients from Wuhan, China. Intensive care Med 46(5): 846-848.

9. Cao X (2020) COVID-19: immunopathology and its 


\section{Open Access Journal of Microbiology \& Biotechnology}

implications for therapy. Nature reviews Immunology 20: $269-70$.

10. Zhang C, Wu Z, Li JW, Zhao H, Wang GQ (2020) The cytokine release syndrome (CRS) of severe COVID-19 and Interleukin- 6 receptor antagonist Tocilizumab may be the key to reduce the mortality. Int J Antimicrob Agent 55(5): 105954.

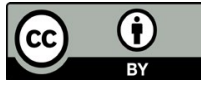

